\title{
An Efficient and Accurate Solution for Distribution System State Estimation with Multi-area Architecture
}

\author{
Marco Pau, Member, IEEE, Ferdinanda Ponci, Senior Member, IEEE, Antonello Monti, Senior Member, IEEE, \\ Sara Sulis, Member, IEEE, Carlo Muscas, Senior Member, IEEE, Paolo Attilio Pegoraro, Member, IEEE
}

\begin{abstract}
Distribution System State Estimation (DSSE) is an essential tool for the management and control of future distribution networks. Distribution grids are usually characterized by a very large number of nodes and different voltage levels. Moreover, different portions of the system can be operated by different Distribution System Operators. In this context, multiarea approaches are key tools to efficiently perform DSSE. This paper presents a novel approach for Multi-Area State Estimation in distribution systems. The proposed algorithm is based on a twostep procedure, where the first-step local estimations are refined through a newly designed second step that allows the integration of the measurement information available in the adjacent areas. The main novelty in this paper is the mathematical analysis of the impact brought by possible measurements shared among different areas, which drives the design of a new efficient Weighted Least Squares formulation of the second step to maximize the achievable estimation accuracy. Tests performed on the unbalanced IEEE 123-bus network prove the goodness of the new multiarea estimator proposed and show the accuracy and efficiency enhancements obtainable with respect to previous literature.
\end{abstract}

Keywords-Distribution System State Estimation, Multi Area State Estimation, Weighted Least Squares, Voltage Estimation, Uncertainty Analysis, Measurement Correlation.

\section{INTRODUCTION}

With the transition of the distribution grids towards more complex and active networks, advanced management and control tools are increasingly required also at distribution level [1]. Distribution System State Estimation (DSSE) can be seen as the core of the Distribution Management System (DMS) envisaged for the control of future distribution grids, since it provides the information on the network operating conditions that is required by most of the control algorithms [2], [3].

In last years, several works faced the problem of state estimation in distribution systems [4]-[7]. Nevertheless, DSSE still remains a challenging task because of several reasons. One of the main issues is the scarcity of measurement devices available on the field. Usually, pseudo-measurements obtained from statistical or historical data are used to make the DSSE problem solvable. However, the reliability for this kind of

M. Pau, F. Ponci, A. Monti are with the Institute for Automation of Complex Power Systems, RWTH Aachen University, Aachen 52062, Germany (email: [amonti, mpau, fponci]@eonerc.rwth-aachen.de)

S. Sulis, C. Muscas, P. A. Pegoraro are with the Department of Electrical and Electronic Engineering of the University of Cagliari, Piazza d'Armi, 09123 Cagliari, Italy (email: [carlo, paolo.pegoraro, sara.sulis]@diee.unica.it).

(c) IEEE 2017. Personal use of this material is permitted. Permission from IEEE must be obtained for all other uses. DOI:10.1109/TIM.2016.2642598. Publisher version: http://ieeexplore.ieee.org/document/7831476/ information is generally very low and this adversely affects the accuracy achievable in the estimation results [8].

Another important problem is the size of the distribution systems. Medium Voltage (MV) grids can have several thousands of nodes and this number would drastically increase if Low Voltage (LV) networks were also considered. The dimension of the grid obviously affects the computational burden of the DSSE algorithm and, consequently, the final execution times. A way to deal with this problem is to use Multi-Area State Estimation (MASE) algorithms. In a multi-area framework, the whole grid is divided in several zones that can be processed indipendently, and this allows simplifying and speeding up the overall estimation process. Furthermore, a MASE approach can be sometimes required also to handle portions of the grid having different features (e.g. different voltage levels) or being operated by different Distribution System Operators (DSOs).

Several MASE approaches have been proposed in the literature, based on different criteria for the network partition, the computing architecture and the coordination scheme [9]-[12]. However, most of them are designed for transmission systems and rely on the high redundancy of measurements generally available in these systems. Because of the peculiarities of the distribution networks, the same approaches are not easily applicable at distribution level and, thus, ad hoc MASE algorithms tailored to DSSE are required. So far, only few works deal with multi-area DSSE. In [13] a scalable approach is used, where a centralized coordination step is used to harmonize the local voltage estimations. In [14], a differential evolution algorithm is presented: local estimators exchange data with the neighbouring areas at each iteration of the algorithm, thus requiring high communication costs and complex coordination. More recently, [15] proposed a multi-area estimator based on an overlapping zone approach. The algorithm uses many zonal interactions for integrating the local estimates and requires the execution of full local area state estimation after each exchange of data with the adjacent areas.

The Authors faced the problem of multi-area DSSE in [16]. Different two-step procedures were analyzed, characterized by in parallel local estimations, low communication requirements, and distributed computation. In [17] the impact of possible correlations existing among local state estimations was investigated and taken into account for enhancing the accuracy performance of the algorithm. Finally, in [18], a new second step was proposed to improve the computational burden of the multi-area estimator. In this paper, a newly designed second step of the MASE algorithm is proposed. The aim is to further improve the accuracy of the estimation results achievable at the 
end of the MASE procedure, while keeping the advantages of [18] in terms of computational speed. The main contribution of this paper is twofold. First, a mathematical analysis has been developed to highlight the detrimental effects on the estimation accuracy given by possible measurements shared among different areas. Second, a modified version of the Weighted Least Squares (WLS) second-step algorithm has been conceived, based on the analytical findings, which removes the causes of accuracy degradation and allows improving the MASE results, while keeping very low computational burden.

In the following, Section II describes the proposed multiarea DSSE approach, underlining the benefits associated to the designed architecture and algorithm. Section III focuses on the design of the second step and shows the impact of the shared measurements on the estimation results as well as the solution conceived to compensate for the errors brought by the correlations resulting in the second step of the MASE process. The proposed algorithm was tested on the 123-bus IEEE distribution network and simulation results are reported in Section IV. Final comments and conclusions are given in Section V.

\section{The Proposed MUlti-AreA DSSE}

Several approaches can be adopted to perform MASE. Solutions can differ mainly due to the type of network partition, computing architecture, coordination scheme and methodology. Depending on the design of the MASE algorithm, different features can be obtained in terms of accuracy, robustness, computational efficiency and amount of data exchange [9]. It is worth noting that in the distribution system scenario these aspects are further emphasized because of the very large size of the distribution grids (causing high execution times and large amount of data communication) and the lack of a suitable measurement infrastructure (leading to the risk of low robustness and poor accuracy for the estimation results). As a result, the MASE design should be evaluated accordingly in order to find the proper trade-off among the conflicting requirements of high computational efficiency, low communication costs, high robustness and high accuracy.

\section{A. Multi-area architecture}

The MASE approach proposed in this paper is designed relying on the following assumptions.

- Each area should have the measurements needed for its observability, also considering the availability of pseudo-measurements. This provides robustness to the MASE because minimum accuracy requirements can be obtained even in case of communication failures by using the first step estimation results.

- An overlapping node is considered between adjacent areas since this allows simplifying the harmonization of the results during the second step [17]. Moreover, the installation of a measurement point in the overlapping nodes, composed of a voltage measurement on the bus and power or current measurements on all the lines converging to that node, is suggested. Even though this measurement point is not mandatory, its presence can bring several benefits (as also indicated in [15]) and allows reaching the observability on the different areas using a lower number of measurement points on the whole grid.

- A decentralized, distributed computing architecture is assumed. Each area would be equipped with its own mini-control center, which is responsible for running the local estimations and for the data communication. Data exchange is required only among adjacent areas.

- The coordination scheme is at state estimation level, meaning that data are exchanged only between the first and the second step of the MASE process. This allows reducing the requirements for data communication and data exchange synchronization.

The grid should be divided in areas having approximatively the same number of nodes. In this way it is possible to avoid the presence of areas acting as bottlenecks during the parallel run of the local estimations, leading to the minimization of the execution times.

\section{B. Multi-area DSSE algorithm}

The proposed multi-area DSSE algorithm is based on a twostep procedure. In the following, the main features of these two steps are described.

1) First step: During the first step, local estimations are performed in parallel in each area. The inputs for each estimator are the real-time, pseudo- and virtual (e.g. zero injections) measurements locally available in the considered area. If a measurement point is installed at the overlapped node, the voltage and the powers on the branches linking that node to other internal buses are directly included in the set of input measurements; instead, the power measurements on lines connecting the overlapping bus to nodes belonging to adjacent zones are summed up to create an equivalent power injection measurement. A similar procedure can be performed also with the measurements from a phasor measurement unit (PMU), in case of synchronized measurement points. In fact, the branch current phasors parting from or converging to the bus can be used to obtain branch currents or equivalent current injections.

The state estimation algorithm used to perform the local estimations is the three-phase Weighted Least Squares (WLS) branch-current estimator presented in [7]. As shown in [19], this choice allows obtaining high computational speed while providing the same accuracy as other WLS formulations. Through the estimator in [7], the direct estimate of a reference bus voltage and of all the branch currents is obtained. Then, the whole voltage profile of the area is calculated, starting from the reference bus voltage, computing the voltage drops. Moreover, the covariance matrix of the estimated states is calculated through the inversion of the estimator Gain matrix. Finally, the uncertainty of the obtained bus voltages is also calculated by applying the law of propagation of uncertainty. As a result, the first step provides as output, for each area, the set of branch-current and node-voltage estimates together with their uncertainty. Common bad data identification functionalities are implemented in each local estimator relying on the classical 
Chi-square method for the detection and the analysis of the largest normalized residual for the identification [20].

2) Second step: The second step aims at integrating, in each area, the measurement information available in the adjacent zones for enhancing the accuracy of the estimation results. As shown in [18], the presence of measurement points in the overlapping nodes allows estimating the branch currents in each area without degrading the achievable accuracy. Indeed, an estimation accuracy equal to that obtainable through a state estimation performed on the whole grid (from now on referred to as Integrated State Estimation, ISE) can be obtained already at the first step. Also when measurement points are not available at the shared nodes, [18] shows that the first step current estimations only have slight accuracy degradation with respect to the ISE results. As a consequence, the second step here proposed focuses on the refinement of the voltage profile, where, conversely, large benefits can be obtained through a suitably designed procedure.

According to the MASE architecture described in Section II-A, each area control center exchanges data with the computing nodes of the adjacent zones. The data needed from each neighbouring zone are the first-step voltage estimations on the shared nodes and their associated uncertainty. Thus, only low communication costs are required for the proposed MASE procedure. The voltage estimations obtained from the adjacent areas are post-processed together with the voltage estimation on the reference bus obtained by the local estimator of the considered area. Additional details on the procedure used for the second step post-processing are provided in the next Section. After the voltage post-processing, a new, improved estimation of the voltage on the chosen reference bus is obtained. Moreover, the uncertainty of such estimation can be also obtained. Then, similarly to the first step, a forward sweep calculation is performed to calculate all the bus voltages and the uncertainty propagation law is applied to obtain the uncertainty of these final estimations. Thus, the final output of the proposed MASE algorithm is the set of currents (obtained at the first step) and voltages (refined through the second step) for each area together with their uncertainties. It is worth noting that, if required by the upper level applications, the power estimates, with their uncertainties, can be also calculated starting from the voltage and current estimates and applying again the law of uncertainty propagation. The flowchart in Fig. 1 shows in a schematic view the summary of the different steps of the MASE process.

\section{SECOND STEP DESIGN}

\section{A. Integration of adjacent area estimations}

The design of the MASE second step is focused on the refinement of the voltage profile estimation. This Section shows how the voltage refinement can be performed through a simple weighted average of voltages. To this purpose, let us consider a branch-current state estimator composed of the following state vector:

$$
\mathbf{x}=\left[v_{r e f}, i_{1}, i_{2}, \ldots, i_{N_{b r}}\right]^{T}
$$

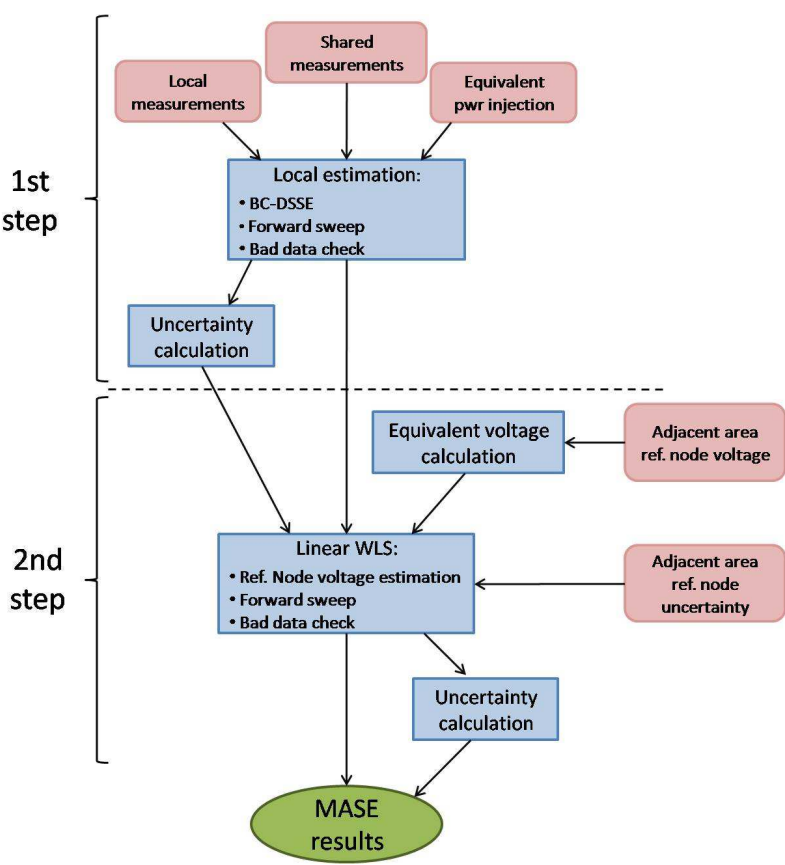

Fig. 1. Flowchart of the proposed MASE.

where $v_{\text {ref }}$ is the voltage phasor on the chosen reference node, $i_{k}$ is the current phasor on the generic $k$-th branch, and $N_{b r}$ is the total number of branches in the considered area.

If a second WLS estimation using the state vector in (1) is performed, and the first step local estimations (both the reference bus voltage and the branch currents) plus the voltages on the overlapping nodes derived by the adjacent areas are used as input measurements, the following linear equation system can be obtained:

$$
\mathbf{G} \cdot \mathbf{x}=\mathbf{H}^{T} \mathbf{W} \cdot \mathbf{y}
$$

where $\mathbf{G}=\mathbf{H}^{T} \mathbf{W H}$ is the WLS Gain matrix, $\mathbf{H}$ is the Jacobian of the measurement functions, $\mathbf{W}$ is the weighting matrix of the measurements, and $\mathbf{y}$ is the following vector of input measurements:

$$
\mathbf{y}=\left[\mathbf{v}^{(1)}, \mathbf{i}^{(1)}\right]^{T}
$$

with $\mathbf{v}^{(1)}$ and $\mathbf{i}^{(1)}$ being the vectors of the real and imaginary parts of the estimates obtained in the first step for the voltages and currents used as input in the second one.

Analyzing the relationship between input measurements and state vector, it is possible to observe that the Jacobian matrix can be decomposed in the following blocks:

$$
\mathbf{H}=\left[\begin{array}{ll}
\mathbf{H}_{v v} & \mathbf{H}_{v i} \\
\mathbf{H}_{i v} & \mathbf{H}_{i i}
\end{array}\right]=\left[\begin{array}{cc}
\mathbf{1} & \mathbf{H}_{v i} \\
\mathbf{0} & \mathbf{I}
\end{array}\right]
$$

where $\mathbf{H}_{v v}$ and $\mathbf{H}_{i v}$ are column vectors (of ones and zeros, respectively) related to the derivatives of the input voltage and current measurements with respect to the state variable $v_{r e f}$, while $\mathbf{H}_{v i}$ and $\mathbf{H}_{i i}$ are the Jacobian sub-matrices associated to the derivatives with respect to the current state variables 
$i_{1}, i_{2}, \ldots, i_{N_{b r}}$. In case of $\mathbf{H}_{i i}$, the corresponding Jacobian submatrix is the identity matrix $\mathbf{I}$, while the terms of $\mathbf{H}_{v i}$ are:

$$
\mathbf{H}_{v i}(j, k)= \begin{cases}-z_{k} & \text { if branch } k \in \Gamma_{j} \\ 0 & \text { if branch } k \notin \Gamma_{j}\end{cases}
$$

where $z_{k}$ is the complex impedance of branch $k$ and $\Gamma_{j}$ is the path between the reference node and the bus associated to the $j$-th input voltage measurement in $\mathbf{v}^{(1)}$.

Since the goal of the second step estimation is the enhancement of the voltage profile, let us focus the attention on the estimation of $v_{r e f}$. Taking into account the first equation in (2) given by the multiplications involving the first row of the Gain matrix $\mathbf{G}$ and the first row of the matrix $\mathbf{B}=\mathbf{H}^{T} \mathbf{W}$, it is possible to find:

$$
g_{11} \cdot v_{r e f}+\sum_{k=2}^{N_{b r}+1} g_{1 k} \cdot i_{k-1}=\sum_{h=1}^{M} b_{1 h} \cdot y_{h}
$$

where $g_{1 k}$ is the $k$-th term of the first row of the Gain matrix, $b_{1 h}$ is the $h$-th term of the first row of matrix $\mathbf{B}, y_{h}$ is the $h$-th measurement in vector $\mathbf{y}$ and $M$ is the total number of input measurements.

Considering the form of the Jacobian in (4), if the voltage measurements $\mathbf{v}^{(1)}$ are decorrelated (the impact given by possible correlations will be analyzed in the next subsections), the following holds:

$$
\begin{gathered}
g_{11}=\sum_{j=1}^{M_{V}} w_{v_{j}} \\
g_{1 k}=-z_{k} \sum_{s \in K} w_{v_{s}} \\
\sum_{h=1}^{M} b_{1 h} \cdot y_{h}=\sum_{j=1}^{M_{V}} w_{v_{j}} \cdot v_{j}^{(1)}
\end{gathered}
$$

where $w_{v_{j}}$ is the weight associated to the $j$-th voltage measurement $v_{j}^{(1)}, M_{V}$ is the total number of the input voltage measurements in vector $\mathbf{y}$, and $K$ is the set of voltage measurements for which branch $k$ is in the path between the reference node and the bus of the voltage measurement.

Considering the findings in (7)-(9), equation (6) can be rewritten as follows:

$$
\sum_{j=1}^{M_{V}} w_{v_{j}} \cdot v_{r e f}=\sum_{j=1}^{M_{V}} w_{v_{j}} \cdot\left(v_{j}^{(1)}+\sum_{k \in \Gamma_{j}} z_{k} i_{k}\right)
$$

From (10), it is evident that the reference bus voltage estimation only depends on the input voltage measurements, their weight and the voltage drops in the paths between the reference node and each of the buses associated to the voltage inputs. This is also in accordance with the analysis performed in [21], where the voltage estimation and its uncertainty were proved to depend on the same quantities.
Let us define now, for each voltage input, the following voltages on the reference node, resulting because of the voltage drops:

$$
v_{j}^{\prime}=v_{j}^{(1)}+\sum_{k \in \Gamma_{j}} z_{k} i_{k}
$$

Using (11) in (10), it is possible to see that the estimation of the voltage on the reference bus reduces to the following weighted average:

$$
v_{r e f}=\frac{\sum_{j=1}^{M_{V}} w_{v_{j}} v_{j}^{\prime}}{\sum_{j=1}^{M_{V}} w_{v_{j}}}
$$

This calculation is equivalent to apply a WLS procedure to estimate the only state variable $v_{r e f}$, using as input the voltage measurements $v^{\prime}$ placed in the reference node (as also done in [18]). Equation (12), to be applied, requires the computation of $v^{\prime}$ and thus the knowledge of the currents $i_{k}$ (see (11)) that are not known a priori. However, in [18] it has been demonstrated that in the proposed MASE approach the current estimates are already accurate at the first step of the procedure. As a consequence, during the second step it is possible to consider $\mathbf{i} \simeq \mathbf{i}^{(1)}$ and to apply (11) and (12) to refine the voltage estimate on the reference bus.

\section{B. Analysis of the error brought by shared measurements}

The results reported in Section III-A were obtained neglecting possible correlations among the voltage measurements and calculating the weighting matrix accordingly. However, as shown in [17], the presence of shared measurement points in the overlapping nodes leads to correlations among the voltage estimates of adjacent areas. This section shows the impact brought by shared measurements on the results achieved in Section III-A.



Fig. 2. Sample network with shared measurement in the overlapping node

To simplify the analysis, let us consider, as an example, the network in Fig. 2. The grid is divided in two areas and has three voltage measurements: the one in node $s$ is shared between areas $A$ and $B$. If the grid is considered as a whole and voltages $V_{R}, V_{S}$ and $V_{T}$ are considered for the voltage estimation, according to (12), the following voltage estimate on the reference node would be obtained:

$$
v_{r e f}=\frac{w_{R} v_{R}^{\prime}+w_{S} v_{S}^{\prime}+w_{T} v_{T}^{\prime}}{w_{R}+w_{S}+w_{T}}
$$

In case of multi-area partition, instead, at the first step area $A$ only processes the voltage measurements in nodes $r$ and $s$, 
while area $B$ uses those in nodes $s$ and $t$. Assuming node $s$ as reference node for both the areas, the following local estimates would be obtained:

$$
\begin{aligned}
& v_{S, A}^{(1)}=\frac{w_{R} v_{R}^{\prime}+w_{S} v_{S}^{\prime}}{w_{R}+w_{S}} \\
& v_{S, B}^{(1)}=\frac{w_{S} v_{S}^{\prime}+w_{T} v_{T}^{\prime}}{w_{S}+w_{T}}
\end{aligned}
$$

where $v_{S, A}^{(1)}$ and $v_{S, B}^{(1)}$ are the local voltage estimates obtained in areas $A$ and $B$, respectively.

The weights associated to these estimates can be calculated and are equal to:

$$
\begin{aligned}
& w_{S, A}^{(1)}=w_{R}+w_{S} \\
& w_{S, B}^{(1)}=w_{S}+w_{T}
\end{aligned}
$$

Using the voltage estimates in (14)-(15) and the weights given in (16)-(17) to refine the voltage estimation in the reference node $s$ through (12), the calculation leads to:

$$
v_{r e f}=\frac{w_{R} v_{R}^{\prime}+2 w_{S} v_{S}^{\prime}+w_{T} v_{T}^{\prime}}{w_{R}+2 w_{S}+w_{T}}
$$

Comparing the result in (18) to the one obtained in (13), it is clear that the shared measurement leads to an error in the final voltage estimate because its contribution is considered multiple times during the two-step procedure.

\section{Compensation of the second step estimation error}

To compensate for the estimation error highlighted in Section III-B, in this paper a new second step procedure has been designed. The objective is to delete the multiple contribution of the shared measurements in the final result. The design of this procedure and the consequent deletion of the estimation error brought by shared measurements is an important improvement with respect to other MASE techniques relying on overlapped areas where this problem is neither mentioned nor considered.

As mentioned in Section III-A, the weighted average obtained in (12) is equivalent to the application of a mini-WLS for estimating the only reference bus voltage $v_{\text {ref }}$ starting from the input voltages $\mathbf{v}^{\prime}$. The same idea is considered here, but to take into account the impact given by the shared measurements, the following modified WLS is proposed:

$$
\mathbf{H}^{T} \mathbf{W}^{\prime} \mathbf{H} \cdot v_{\text {ref }}=\mathbf{H}^{T} \mathbf{W}^{\prime} \cdot \mathbf{y}^{\prime}
$$

where:

$$
\begin{gathered}
\mathbf{y}^{\prime}=\left[\mathbf{v}^{\prime}, \mathbf{v}_{\text {shar }}\right]^{T} \\
\mathbf{W}^{\prime}=\left[\begin{array}{cc}
\mathbf{W}_{\mathbf{v}^{\prime}} & 0 \\
0 & -\mathbf{W}_{\mathbf{v}_{\text {shar }}}
\end{array}\right]
\end{gathered}
$$

where $\mathbf{v}^{\prime}$ is the vector of voltage inputs reported to the reference node through (11), $\mathbf{v}_{\text {shar }}$ is the vector of all the voltage measurements shared between the considered area and the adjacent ones, and $\mathbf{W}_{\mathbf{v}^{\prime}}$ and $\mathbf{W}_{\mathbf{v}_{\text {shar }}}$ are the corresponding weights obtained as the inverse of their variance. It is worth noting that, since all the measurements in $\mathbf{y}^{\prime}$ are placed at the reference node, the Jacobian $\mathbf{H}$ is simply equal to a column vector of ones.

The application of the above modified WLS formulation allows deleting the multiple contribution of the shared measurements and achieving the same estimation that would be obtained if the considered area and the adjacent ones were considered as a whole. Referring to the same example as in Section III-B, the input measurements $\mathbf{v}^{\prime}$ and their weights $\mathbf{W}_{\mathbf{v}^{\prime}}$ would be given by the quantities in eq. (14)-(17), while the shared measurement and its weight would be $v_{s}$ and $w_{s}$. It is easy to verify that applying (19) the same results as in (13) would be obtained.

The method also works if more than two areas share the same measurement (in this case the measurement has to be included in $\mathbf{y}^{\prime}$ as many times as the number of adjacent areas with which it is shared) and if there are different overlapping nodes with different neighbouring zones.

\section{Proposed second step procedure}

The final design of the proposed MASE second step exploits the results shown in the previous subsections. The procedure is based on the following steps:

- The local voltage estimate on the reference bus, the voltage estimates on the overlapping nodes provided by each adjacent area, and all the voltage measurement on the overlapping nodes (if present) are used as input; all the voltage inputs are acquired together with their variance.

- If the measurement system is not based on PMUs, for each voltage input, the voltage phase-angle calculated at the first step from the local estimator of the considered area on the node associated to the voltage measurement is used to obtain voltage phasors (this is done because of the use of non-consistent reference phase-angles in the different areas of the grid).

- All the voltage inputs are transformed in equivalent voltages on the reference node by using (11). If the measurement system is based on traditional (non-synchronized) measurements, the voltage magnitudes resulting on the reference node are considered for the next step; in case of PMU-based measurement system, both voltage magnitudes and phase-angles are instead taken into account.

- The WLS calculation proposed in (19) is used to estimate the new voltage on the reference node. Similarly to the classical WLS approach, the inverse of the Gain matrix is used to achieve the variance of the new voltage estimate.

- The whole voltage profile of the grid is obtained, starting from the new voltage estimate on the reference node, by considering the voltage drops given by the currents estimated in the first step. The uncertainty propagation law is used to calculate the uncertainty of each node voltage.

- If needed by the upper level applications, the power estimates, together with their uncertainty, can also be calculated using the voltage and current estimates and applying the uncertainty propagation law. 


\section{TESTS AND RESULTS}

\section{A. Test Assumptions}

The proposed multi-area DSSE algorithm was tested on the unbalanced IEEE 123-bus network [22] using a partition in four areas as depicted in Fig. 3. Several tests were performed, using different measurement systems, to highlight the accuracy performance of the proposed MASE method. Tests here presented refer to the following measurement scenarios:

- Case 1: measurement points only in the primary substation and the overlapping nodes (nodes 149, 18, and 67).

- Case 2: measurement points in both overlapping and non-overlapping nodes (nodes 149, 18, 25, 52, 67, 76, 97).

- Case 3: measurement points only in non-overlapping nodes (nodes 149, 25, 52, 76, 97).

In all the cases each measurement point is considered to be composed of a voltage measurement at the bus and the monitoring of the power (in case of traditional measurement system) or the current (in case of PMU-based measurement system) in all the branches converging to the node.

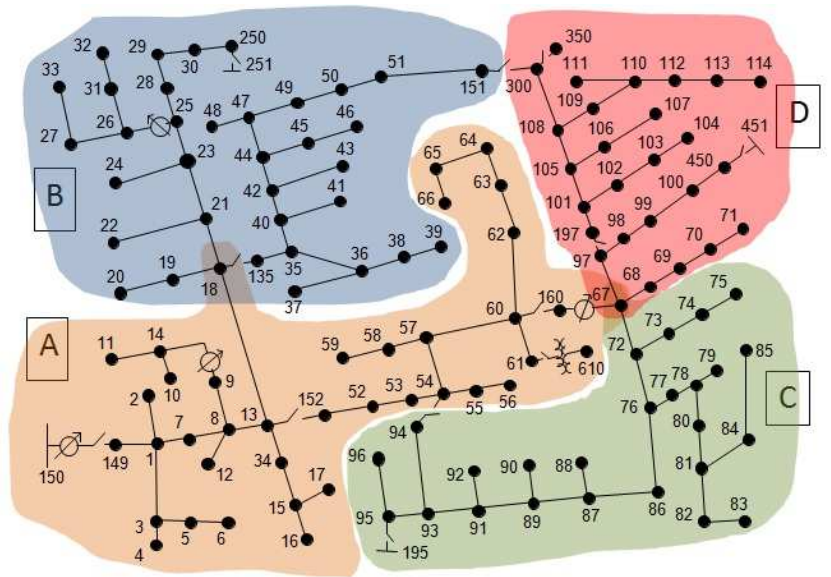

Fig. 3. IEEE 123-bus test network

To evaluate the accuracy performance of the proposed method, tests were performed by using Monte Carlo (MC) simulations. For each simulation, at the beginning, the reference values of the electrical quantities in the network were obtained by means of a load flow calculation. Then, for each MC trial, measurements were randomly extracted by perturbing the reference values according to the assumed measurement uncertainty. In particular, in all the presented tests, the following assumptions were considered.

- Number of MC trials: $N_{M C}=25000$.

- Pseudo-measurements of bus power injection with Gaussian distributed error and deviation $3 \sigma=50 \%$ of the nominal value.

- Measurements with Gaussian distributed error and $3 \sigma$ equal to the measurement accuracy. As for traditional measurements, accuracies equal to $1 \%$ and $3 \%$ of the reading value are used for voltage magnitude and power

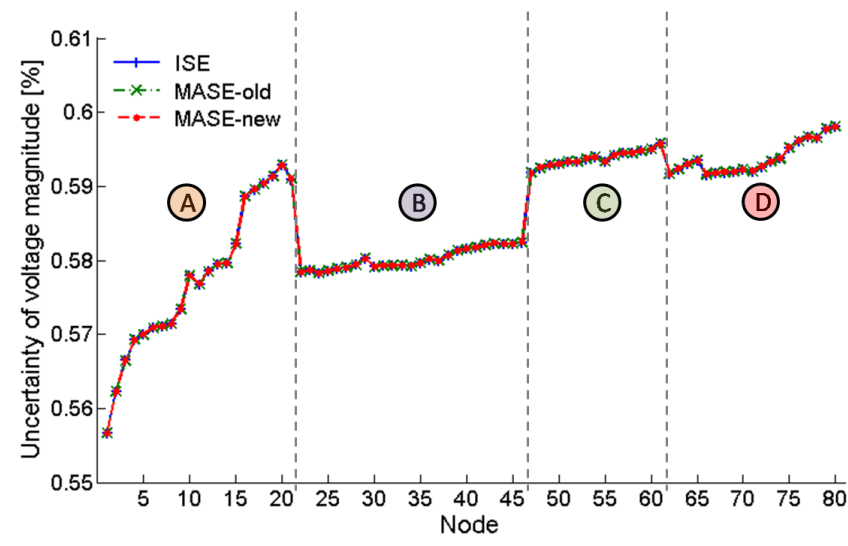

Fig. 4. Uncertainty of voltage magnitude estimation with Case 1 measurement system (traditional measurements).

measurements, respectively. In case of PMUs, accuracies of $0.7 \%$ and $0.7 \mathrm{crad}\left(0.7 \cdot 10^{-2} \mathrm{rad}\right)$ are considered for magnitude and phase-angle measurements, respectively. It is worth noting that the values chosen here are only an example of possible accuracies, but in the real scenarios the measurement accuracy has to be properly evaluated, considering also the transducers and the other components of the measurement chain, in order to assign the correct weights [23].

\section{B. Results}

To show the benefits associated to the new formulation of the multi-area DSSE procedure (MASE-new), in the following, the results are compared to those obtained through the Integrated State Estimation (ISE), which is the estimation carried out on the whole network, and to those achievable through the multiarea method proposed in [17] (MASE-old), which provides the best reference in terms of accuracy results (since the proposal in [18] was conceived only to improve the computational performance). It is worth noting that multi-area techniques usually lead to some degradation in the final accuracy, because not all the measurements available in the grid can be processed simultaneously. As a consequence, ISE results can be considered as the reference optimum estimation achievable through the given set of available measurements. As for MASE-old, this procedure is based on a two-step approach where a whole state estimation is performed also at the second step using as input the previous local estimates (the full state vector), the local zero injections and the boundary estimates provided by the adjacent areas. This procedure also considers (in the weighting matrix) the correlations between the estimates of the considered area and the inputs coming from the adjacent zones. Therefore, it represents a meaningful term of comparison for evaluating benefits and drawbacks associated to the new MASE formulation in terms of accuracy of the results.

First, a test has been performed by considering the Case 1 measurement system. This configuration represents a particular scenario because all the available measurements are included in one of the areas (area A). In this case, the MASE procedure can 
achieve the same results as the ISE. In fact, the measurement point at the overlapping nodes allows properly estimating all the branch currents already at the first step (same accuracy as ISE in each area, as already described in [18]), while the second step permits propagating the accuracy of the voltage estimation in area A to all the other zones. Fig. 4 shows the expanded uncertainties (with a coverage factor equal to 3) obtained for the voltage magnitude estimation of phase A when a traditional measurement system is considered (here and in the following tests, same considerations also apply to all the other phases of the system). It is possible to observe that the new design of the second step allows properly compensating the errors arising due to the shared measurements, similarly to what is obtained in MASE-old by considering the correlations in the weighting matrix. However, it is worth remarking that the same accuracy results are achieved at the expense of a much lower computational burden: indeed, MASE-old implies the repetition of a full grid SE process (at the second step of each area) while MASE-new only requires a simple, linear second step aimed at the estimation of the reference bus voltage. In the tests here presented, considering area $\mathrm{A}$ and a traditional measurement system as example, this means that, for MASE-old, an iterative SE process involving 135 state variables and more than 200 measurements is needed, while MASE-new only requires the equivalent voltages computation in (11) and a linear WLS process including, per each phase, 1 state variable (the reference bus voltage) and 7 measurements (4 voltage inputs plus 3 shared voltage measurements). In this example, this results in more than 7.5 MFLOPs at each iteration of MASE-old (using sparse matrices) only for the solution of the WLS normal equations, while less than 0.3 kFLOPs are needed in MASE-new for both the equivalent voltages calculation and the solution of the linear WLS. These numbers also provide an idea of the advantages of the proposed algorithm with respect to other MASE proposals available in the literature and based on multiple zonal interactions, where the whole area DSSE can be repeated also a large number of times before reaching the prefixed convergence threshold (see for example [15]). Results similar to those of Fig. 4 can be also found when using a PMU-based measurement system. The corresponding graphs are not reported here for space reasons.

Further benefits associated to the new MASE procedure can be found when considering a more complex system with more measurements in each area in addition to those in the overlapping nodes. In this case, again, the presence of the measurements at the shared nodes allows having good current estimations already at the first step (same accuracy as ISE). As for the voltages, the accuracy level is strictly depending on the number of voltage measurements that are processed through the two steps of the MASE process. Fig. 5 shows the voltage magnitude expanded uncertainties obtained in case of traditional measurements when considering the configuration in Case 2. First of all, it is possible to note that in this case the same accuracy as ISE cannot be obtained in all the areas. However, the newly proposed MASE procedure leads to evident improvements in terms of accuracy with respect to MASE-old. In particular, in area A, the same accuracy as ISE can be achieved, since all the voltage measurements of the

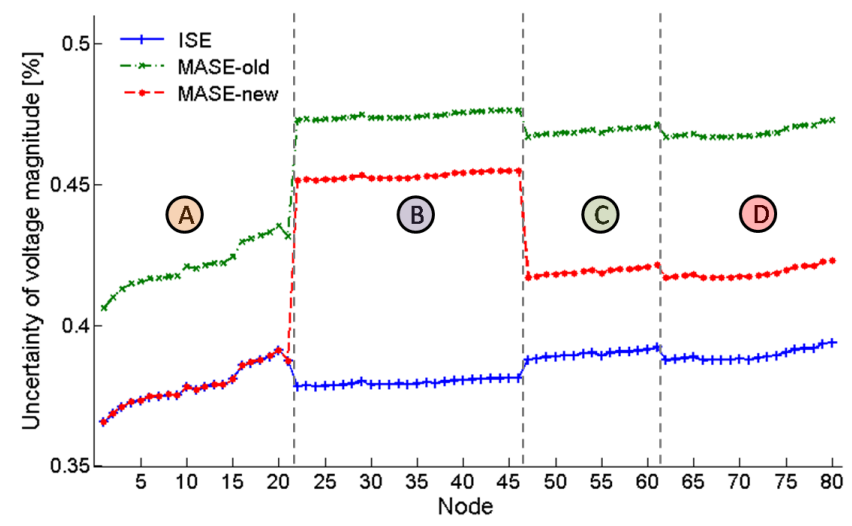

Fig. 5. Uncertainty of voltage magnitude estimation with Case 2 measurement system (traditional measurements).

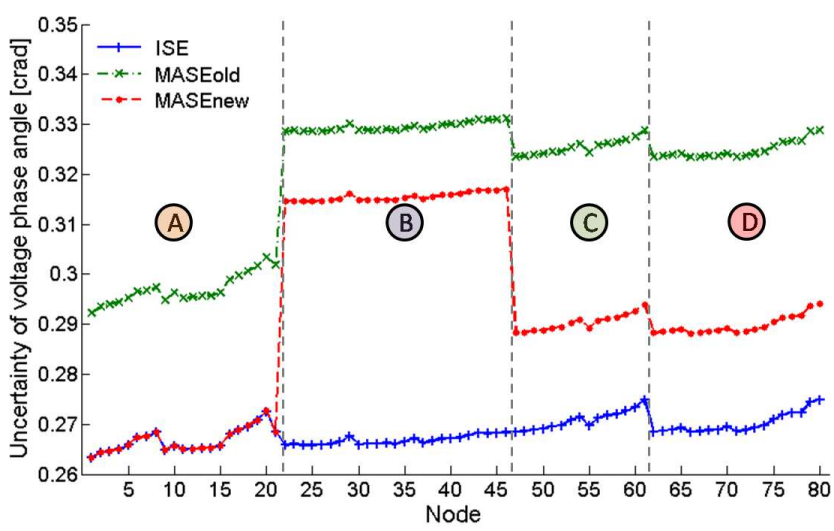

Fig. 6. Uncertainty of voltage phase-angle estimation with Case 2 measurement system (PMU measurements)

grid are incorporated through the acquisition of the voltage estimates coming from the neighbouring areas. In all the other areas, even though the accuracy is worse than ISE, the second step of MASE-new outperforms MASE-old, proving that the new approach is not only more efficient from a computational point of view but also more suitable to avoid the detrimental effects of the shared measurements on the accuracy.

Results shown in Fig. 5 for MASE-new are also in agreement with the voltage uncertainty expected according to [21], depending on the total number of processed voltage measurements (note that voltage measurement uncertainty is equal to $1 \%$ of the reading value): indeed, area $\mathrm{A}$ has a voltage uncertainty around $0.38 \%$, which is related to 7 voltage measurements (4 measurements in A plus 3 coming from B, $\mathrm{C}$ and $\mathrm{D})$, area $\mathrm{B}$ has a voltage uncertainty around $0.45 \%$, which corresponds to 5 measurements (2 local measurements in $\mathrm{B}$ plus 3 derived from $\mathrm{A}$ ) and areas $\mathrm{C}$ and $\mathrm{D}$ have voltage uncertainty approximatively equal to $0.41 \%$, which is associated to 6 measurements ( 2 locally available, plus 4 acquired from the neighbouring zones).

Similar results and considerations have been obtained also in case of measurement system composed of PMUs. It is worth noting that in this case, the same considerations made for 


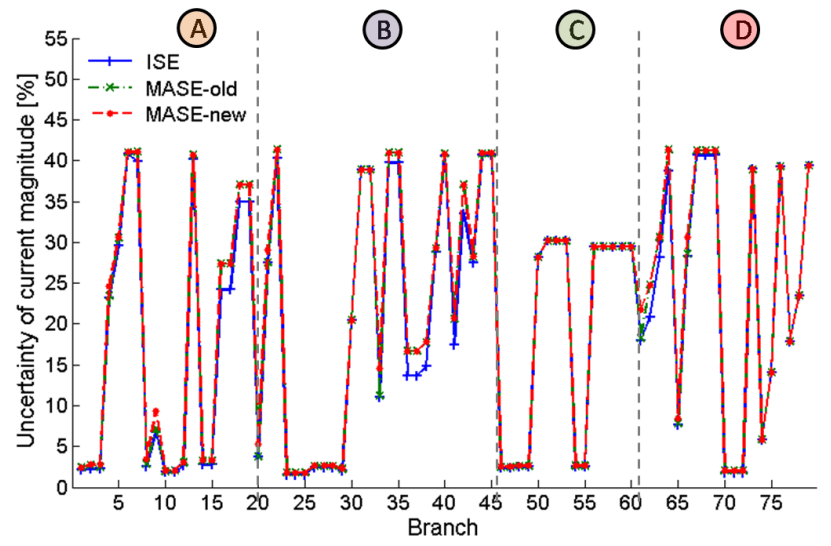

Fig. 7. Uncertainty of current magnitude estimation with Case 3 measurement system (traditional measurements).

the voltage magnitude estimation also apply to the voltage phase-angle estimation. Fig. 6 shows the expanded uncertainties in the voltage phase-angle estimates obtained with the Case 2 measurement scenario. It is possible to notice that improvements analogous to those in Fig. 5 are obtained and the same considerations on the expected uncertainty levels, depending on the number of processed voltage synchrophasor measurements [24], can be drawn.

As last test scenario, the measurement configuration described in Case 3 is considered. In this case, no measurements are available in the overlapping nodes and, therefore, all the issues related to the presence of shared measurements in the different areas are avoided. Even if the use of measurements in the shared nodes can provide several benefits, this test allows evaluating the possible limits of the presented MASE approach and to assess the capability of the proposed method to work with any measurement configuration. As already described in [18], the main drawback given by the lack of measurement points in the overlapping nodes is the slight degradation of the estimation accuracy for the currents in the branches close to the border node. Fig. 7 shows, as an example, the obtained expanded uncertainty of the current magnitude estimates in case of a traditional measurement system. As it can be observed, the new MASE proposal has a slight worsening of the boundary current estimations since no current estimation refinement is performed during the second step. However, this degradation is local and only concerns few branches adjacent or close to the overlapping node.

Fig. 8 shows instead the effects of this degradation on the uncertainty of the voltage magnitude estimates. The obtained results clearly shows that, since the impact of the current estimation degradation is local, only very small effects can be detected on the voltage estimates. In this case, MASE-new and MASE-old exhibit similar performance and, again, the uncertainty of the voltage profile can be approximatively inferred by the total number of voltage measurements processed during the two steps of the MASE approach.

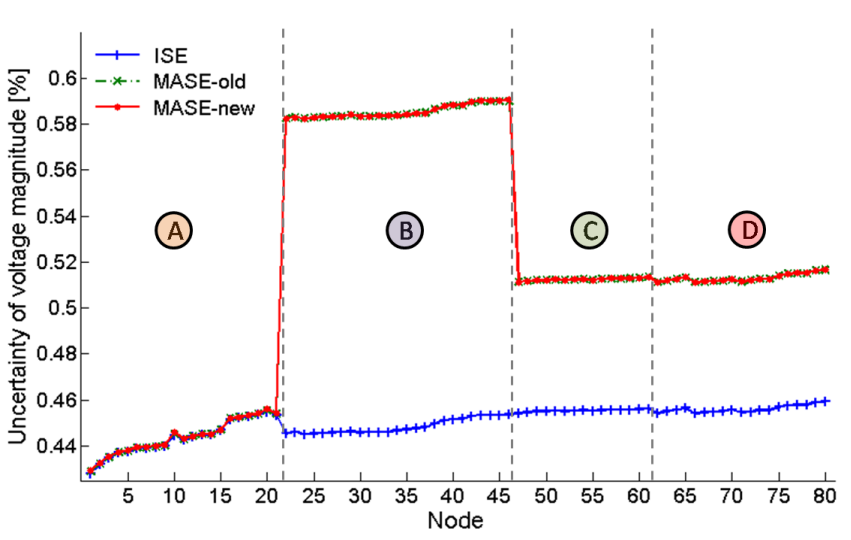

Fig. 8. Uncertainty of voltage magnitude estimation with Case 3 measurement system (traditional measurements).

\section{Communication requirements}

Some considerations on the communication issues of a possible DSSE architecture are discussed in the following for the case of a PMU-based architecture. A PMU output stream depends on the reporting rate and on the encapsulation protocol. Considering, for example, a TCP/IP packetization (44 bytes as in [25]) and a PMU working at $50 \mathrm{fps}$ (50 Hz system) with 9 channels ( 3 three-phase channels), as those considered in the tests, the output bandwidth can range from a minimum of $40.8 \mathrm{kbps}$ for 16-bit fixed point format to $85.6 \mathrm{kbps}$ for floating point representation and additional positive, negative and zero sequence phasors. These values are a lower bound for a PMU bandwidth, since PMU packets can also host further analog and digital data (see [25] for details) and the possibility to have 100-120 fps is encouraged by [26]. Besides, larger headers can be present due to the chosen protocol architecture, while configuration and command frames have been neglected here for the sake of simplicity. Such bitrates are not easily manageable, for example, by narrowband power line communications [27] adopted for automatic meter reading, advanced metering infrastructure and other applications. Several pioneer projects are ongoing to define full communication infrastructures for MV/LV DNs, that are expected to be hybrid [28] (power line, wireless and optical fiber technologies) and hierarchical [29].

In ISE a single step is present and all the PMU data must be received by the control center. The overall bandwidth depends on the total number of devices. In MASE algorithms, instead, the bitrate is divided following the division into areas and second-step data exchange is between control centers of adjacent areas. The main advantage of MASE-old and MASEnew techniques is that the second step requires a limited data exchange at the DSSE rate, thus reducing synchronization and latency issues. MASE-new further reduces the exchanged data because it only transfers voltage estimation between adjacent areas.

The latency requirements of the monitoring systems strongly depend on the application needs: the overall latency can range from tens of milliseconds for fault location isolation and service restoration to seconds or tens of seconds for voltage regulation update. To give another example, in [30], the 
transfer time associated to message performance classes goes from milliseconds to seconds depending on the applications. For this reason, in SG perspective, the latency due to the DSSE and the corresponding communications, which is just a portion of the overall management system latency, must be kept as small as possible to build on the monitoring activity as many different applications as needed. In [31], for instance, the implemented prototype of a real-time DSSE system works at $1 \mathrm{~Hz}$ with PMUs at $4 \mathrm{fps}$. Obviously, the coordination of heterogonous measurement rates suggests different possible solutions that are beyond the scope of this paper.

\section{CONCLUSIONS}

In this paper, an efficient and accurate solution for multi-area state estimation in distribution grids has been presented. The proposed formulation relies on a modified version of the WLS approach, which allows efficiently including the information provided by the neighbouring zones and properly integrating the measurements shared among the different areas. Results obtained on the IEEE 123-bus network show the benefits associated to the proposed methodology in terms of accuracy and computational efficiency. The proposed multi-area estimator design also allows using a distributed architecture with minimum communication costs. Thus, it provides a fine trade-off among the conflicting requirements of high accuracy and robustness, and low computation and communication requirements. The results of this study can serve as a basis for further works on the design of distributed architectures for the automation and accurate monitoring of distribution grids.

\section{REFERENCES}

[1] J. Fan and S. Borlase, "The evolution of distribution," Power and Energy Magazine, IEEE, vol. 7, no. 2, pp. 63-68, March 2009.

[2] A. Meliopoulos, E. Polymeneas, Z. Tan, R. Huang, and D. Zhao, "Advanced distribution management system," Smart Grid, IEEE Transactions on, vol. 4, no. 4, pp. 2109-2117, Dec 2013.

[3] G. Celli, P. A. Pegoraro, F. Pilo, G. Pisano, and S. Sulis, "DMS cyberphysical simulation for assessing the impact of state estimation and communication media in smart grid operation," IEEE Trans. Power Syst., vol. 29, no. 5, pp. 2436-2446, Sep. 2014.

[4] M. E. Baran and A. W. Kelley, "State estimation for real-time monitoring of distribution systems," IEEE Trans. Power Syst., vol. 9, no. 3, pp. 1601-1609, Aug. 1994

[5] M. Baran and A. Kelley, "A branch-current-based state estimation method for distribution systems," IEEE Trans. Power Syst., vol. 10, no. 1, pp. 483-491, Feb. 1995.

[6] A. Cataliotti, V. Cosentino, D. D. Cara, and G. Tin, "LV measurement device placement for load flow analysis in MV smart grids," IEEE Trans. Instrum. Meas., vol. 65, no. 5, pp. 999-1006, May 2016.

[7] M. Pau, P. A. Pegoraro, and S. Sulis, "Efficient branch-current-based distribution system state estimation including synchronized measurements," IEEE Trans. Instrum. Meas., vol. 62, no. 9, pp. 2419-2429, Sep. 2013.

[8] A. Angioni, T. Schlsser, F. Ponci, and A. Monti, "Impact of pseudomeasurements from new power profiles on state estimation in lowvoltage grids," IEEE Transactions on Instrumentation and Measurement, vol. 65, no. 1, pp. 70-77, Jan 2016.

[9] A. Gomez-Exposito, A. de la Villa Jaen, C. Gomez-Quiles, P. Rosseaux, and T. Van Cutsem, "A taxonomy of multi-area state estimation methods," Electric Power Systems Research, vol. 81, no. 4, pp. 1060-1069, Apr. 2011.
[10] D. Falcao, F. Wu, and L. Murphy, "Parallel and distributed state estimation," Power Systems, IEEE Transactions on, vol. 10, no. 2, pp. 724-730, May 1995.

[11] M. Zhao and A. Abur, "Multi area state estimation using synchronized phasor measurements," Power Systems, IEEE Transactions on, vol. 20, no. 2, pp. 611-617, May 2005.

[12] A. Conejo, S. De La Torre, and M. Canas, "An optimization approach to multiarea state estimation," Power Systems, IEEE Transactions on, vol. 22, no. 1, pp. 213-221, Feb. 2007.

[13] L. De Alvaro Garcia and S. Grenard, "Scalable distribution state estimation approach for distribution management systems," in Innovative Smart Grid Technologies (ISGT Europe), 2011 2nd IEEE PES International Conference and Exhibition on, Dec. 2011, pp. 1-6.

[14] N. Nusrat, M. Irving, and G. Taylor, "Development of distributed state estimation methods to enable smart distribution management systems," in Industrial Electronics (ISIE), 2011 IEEE International Symposium on, Jun. 2011, pp. 1691-1696.

[15] N. Nusrat, P. Lopatka, M. R. Irving, G. A. Taylor, S. Salvini, and D. C. H. Wallom, "An overlapping zone-based state estimation method for distribution systems," IEEE Transactions on Smart Grid, vol. 6, no. 4, pp. 2126-2133, July 2015.

[16] C. Muscas, M. Pau, P. A. Pegoraro, S. Sulis, F. Ponci, and A. Monti, "Two-step procedures for wide-area distribution system state estimation," in Instrumentation and Measurement Technology Conference (I2MTC), 2014 IEEE International, May 2014, pp. 1517-1522.

[17] C. Muscas, M. Pau, P. A. Pegoraro, S. Sulis, F. Ponci, and A. Monti, "Multiarea distribution system state estimation," IEEE Transactions on Instrumentation and Measurement, vol. 64, no. 5, pp. 1140-1148, May 2015.

[18] C. Muscas, P. A. Pegoraro, S. Sulis, M. Pau, F. Ponci, and A. Monti, "Fast multi-area approach for distribution system state estimation," in Instrumentation and Measurement Technology Conference (I2MTC), 2016 IEEE International, May 2016.

[19] M. Pau, P. A. Pegoraro, and S. Sulis, "Performance of three-phase WLS distribution system state estimation approaches," in Applied Measurements for Power Systems (AMPS), 2015 IEEE International Workshop on, Sept 2015, pp. 138-143.

[20] A. Abur and A. G. Expòsito, Power System State Estimation. Theory and Implementation. Marcel Dekker, New York, 2004.

[21] M. Pau, P. A. Pegoraro, S. Sulis, and C. Muscas, "Uncertainty sources affecting voltage profile in distribution system state estimation," in 2015 IEEE International Instrumentation and Measurement Technology Conference (I2MTC) Proceedings, May 2015, pp. 109-114.

[22] (2012) IEEE test feeder specifications. [Online]. Available: http: //ewh.ieee.org/soc/pes/dsacom/testfeeders/

[23] M. Asprou, E. Kyriakides, and M. Albu, "The effect of variable weights in a wls state estimator considering instrument transformer uncertainties," IEEE Trans. Instrum. Meas., vol. 63, no. 6, pp. 14841495, Jun. 2014.

[24] C. Muscas, M. Pau, P. A. Pegoraro, and S. Sulis, "Uncertainty of voltage profile in PMU-based distribution system state estimation," IEEE Transactions on Instrumentation and Measurement, vol. 65, no. 5, pp. 988-998, May 2016.

[25] IEEE Standard for Synchrophasor Data Transfer for Power Systems, IEEE Std C37.118.2-2011 (Revision of IEEE Std C37.118-2005), Dec. 2011.

[26] IEEE Standard for Synchrophasor Measurements for Power Systems, IEEE Std C37.118.1-2011 (Revision of IEEE Std C37.118-2005), Dec. 2011 .

[27] G. Artale, A. Cataliotti, V. Cosentino, D. D. Cara, R. Fiorelli, P. Russotto, and G. Tin, "Medium voltage smart grid: Experimental analysis of secondary substation narrow band power line communication," IEEE Transactions on Instrumentation and Measurement, vol. 62, no. 9, pp. 2391-2398, Sept 2013.

[28] D. D. Giustina and S. Rinaldi, "Hybrid communication network for the 
smart grid: Validation of a field test experience," IEEE Trans. Power Del., vol. 30, no. 6, pp. 2492-2500, Dec. 2015.

[29] S. Lu, S. Repo, D. D. Giustina, F. A. C. Figuerola, A. Lf, and M. Pikkarainen, "Real-time low voltage network monitoring - ICT architecture and field test experience," IEEE Trans. Smart Grid, vol. 6, no. 4, pp. 2002-2012, Jul. 2015.

[30] Communication networks and systems in substations Part 5: Communication requirements for functions and device models, IEC Std. 61850-5, Apr. 2013.

[31] A. Angioni, J. Shang, F. Ponci, and A. Monti, "Real-time monitoring of distribution system based on state estimation," IEEE Trans. Instrum. Meas., vol. 65, no. 10, pp. 2234-2243, Oct. 2016. 